\title{
Complex First? On the Evolutionary and Developmental Priority of Semantically Thick Words
}

\author{
Markus Werning
}

When one conjoins relatively well supported views on language acquisition and typology with frequently held views on the neural realization of meaning and some general principles of evolution and development, one seems to arrive at what I shall call the Complex-First Paradox. ${ }^{1}$ At its core is the question why concepts of substances, typically expressed by concrete nouns, seem to lexicalize ontogenetically and phylogenetically so early, even though they are apparently semantically far more complex than concepts that lexicalize later. The paradox consists of five propositions each of which seems plausible in its own right and is supported by empirical or theoretical reasons. The set of propositions - as is the nature of paradoxes - is apparently inconsistent, though, and thus points to an explanatory deficit in linguistic theory:

(P1) The meanings of concrete nouns, in ontogeny and (probably) phylogeny, are acquired earlier than those of many - eventually even all - other word classes.

(P2) The meanings of concrete nouns are substance concepts.

(P3) Substance concepts are semantically more complex and their neural realizations more widely distributed in cortex than those expressed by the other word classes in question.

(P4) For a cortically implemented syntax-semantics interface, the more widely distributed a concept's neural realization is, the more effort it takes to establish a link between the concept and some lexical expression thereof.

(P5) In ontogeny and phylogeny, capabilities demanding more effort, all other things being equal, develop and, respectively, evolve later than those demanding less effort.

The paradox should be obvious now: Assume that the meanings of concrete nouns like daddy, water, and cat are indeed semantically more complex or, to use another word, thicker than the meanings of other word classes, e.g., adjectives like blue, big, and bold. If one accepts that meanings are mental concepts, the view is illustrated as follows: The substance concept [water] has not only perceptual components of various modalities like [transparent], [fluid], and [tasteless], but also components that relate to affordances like [to drink]. The attributive concept [blue], in contrast, seems to be relatively thin: it does not decompose into distinct conceptual parts and seems to pertain to the visual domain only.

\footnotetext{
${ }^{1}$ I have first coined this notion in Werning (2003) as a comment on Arbib (2005). An extended discussion oft he paradox has been presented in Werning (2008).
} 
Assume, furthermore, that conceptual complexity correlates with a wider distribution of the conceptual parts, respectively, their neural realizations in the cortex. One then expects the neural correlate of [water] to pertain to visual, tactile, gustatory, and action-related regions. In contrast, the correlate of [blue] seems to be bound to the visual cortex.

Following another of the assumptions, a word-to-meaning assignment ought to be more easily tractable for a cortically realized syntax-semantics interface if the neural correlate of the meaning is relatively local, rather than widely distributed. Consequently, the link between the adjective blue and the attributive concept [blue] should require less effort than the link between water and [water].

Take it as a quite general principle of evolution now that with regard to one and the same domain incrementally more complex capabilities ceteris paribus evolve later than simpler ones. There had to be feathers first, only then some reptile species could evolve wings. Vision could succeed in evolution only after light-detection had evolved. It seems to be a simple truth that lies behind it: Natura non facit saltus. There is an outright analogy in development: A child must have acquired the capability to hold a stick before it will be able to use a hammer. Children have to acquire simple closed syllables (CVC, e.g., come) before they are able to pronounce syllables with complex codas (CVCC, e.g., cast).

Given those assumptions, how can it be that the meaning of the noun water ontogenetically and phylogenetically still is acquired earlier than that of the adjective blue? Since the concept [water] is semantically more complex than [blue], its neural correlate should be more widely distributed, the link between the concept and its expression should imply more effort, and thus ought to be established later in ontogeny and phylogeny. Rather than the empirical claim made by the first proposition, we should on the basis of the other four assumptions expect that the meanings of concrete nouns, in ontogeny and phylogeny, be acquired later than those of other word classes. In the paper I would like to press the paradox a little further by putting forward arguments for each of the five propositions and rejecting objections against them. Even though my résumé will be rather pessimistic, I will conclude with some more speculative remarks on a potential solution.

\section{Words and Concepts}

The primary role for concepts is the integration of perception and action control. In order to survive in a world with a multitude of things, subjects must subsume them under concepts. Categorization allows the subject to recognize objects and events in the world as well as states of the body, to generate generalizations, and to preserve this information over time. Only thus goal directed interaction between one's body and the world is possible to the degree we observe it in many species.

With regard to humans, concepts are assigned a twofold explanatory role: (i) as content providers and (ii) as meaning providers. In their first role concepts provide contents to intentional states. In their second role concepts are identified with the meanings of linguistic expressions. Concept are apt to fulfill the two roles because they are individuated as internal states of the system that essentially bear a causalinformational relation of co-variation to external contents (Fodor, 1992). This way, concepts may explain why intentional states are about things and why the meanings of expressions in a given context determine which things are referred to.

Intentional states include such diverse modes as perception, belief, desire, memory, expectation, imagination, emotion, and the will. Concepts provide the satisfaction conditions of intentional states, enter into inferential relations, and play a role in the causation of action. The twofold role of concepts suggests a view that intimately links 
meaning to intentionality. A unified approach of meaning and intentional content holds that the meaning of the sentence water is in the bath tub, the perception of water being colorless, the belief that water boils at $100^{\circ} \mathrm{C}$, and the desire to drink a glass of water have one thing in common: they involve the concept [water]. This at least follows if one assumes (i) the compositionality of linguistic meaning, and (ii) the compositionality of intentional content. The compositionality of meaning is the principle that the meaning of a complex expression is in a syntax-dependent way determined by the meanings of its parts. It explains how the concept [water] contributes to the meaning of the sentence water is in the bath tub. The compositionality of content says that the content of a complex intentional state is in a structure-dependent way determined by the contents of its parts. It explains how the content of the concept [water] determines the contents of the perceptual, doxastic, and volitional states just mentioned (for a discussion of the reasons for compositionality see Werning, 2005b).

In our context, the most important distinction in the domain of concepts is that between attributive concepts and substance concepts. Attributive concepts represent features of objects that are volatile in the sense that one and the same object can fall under different attributive concepts at different times: An object may, e.g., change its color, size, or speed, but still continues to exist. [blue] thus is a paradigmatic attributive concept.

Substance concepts, in contrast, are governed by the identity conditions of objects: A mug ceases to exist when it no longer falls under the substance concept [mug], say, because it has been shattered. Substance concepts serve to re-identify things over time in spite of their contingent changes of attributes and so allow us to gather, store and update information in a systematic and enduring way (Millikan, 1998). They are typically expressed by concrete nouns. Attributive concepts, in contrast, are typically expressed by adjectives or abstract nouns.

\section{Nouns and Adjectives}

The paradox arises from the fact that substance concepts are ontogenetically and probably phylogenetically earlier lexicalized than attributive concepts. The great mass of children's earliest words are concrete nouns. During the so-called naming explosion, when children around 18 months of age first systematically organize their concepts by means of a lexicon, they preponderantly pair substance concepts with concrete nouns, whereas the assignment of adjectives and abstract nouns to the attributive concepts they express comes much later (Ingram, 1989). Some languages even don't have adjectives or just a closed set of them (Dixon, 1999), while the class of concrete nouns is arguably universal and always open. One may thus also argue that nouns in phylogeny are prior to adjectives. With respect to the typology of the earliest words, Barrett (1995) in a handbook article provides the following overview:

0th-100th word: high proportion of common nouns.

200th-..: proportion of common nouns decreases.

50th-100th word: proportion of verbs begins to increase.

400th-500th word: verb proportion continues to increase and finally begins to level out.

50th-100th word: proportion of adjectives begins to increase.

100th-500th word: proportion of adjectives continues to increase. 
Even authors like Bloom (2000) who are more critical of the notion of a naming explosion concede that in the earliest phase of language development there is an "object bias": A new word by default is interpreted as a name of an object (i.e., as a concrete noun). It needs some counterevidence for the child to realize that a word (an adjective or verb) expresses a property or an action, instead.

If the data are interpreted correctly, we can make the following inference: Since concrete nouns express substance concepts and prototypical adjectives express attributive concepts, and since concrete nouns are earlier acquired by the child than adjectives, it logically follows that substance concepts are ontogenetically earlier lexicalized than attributive concepts.

With respect to the claim on phylogeny, the evidence is more indirect and less compelling - hence the qualification "probably". It is an undenied fact that in all languages, in which the types of nouns and adjectives exist, there are more concrete nouns than adjectives (Dixon, 1999). Even in English (Givon, 1970) most adjectives are derived from either nouns or verbs, while there are only very few original adjectives. One can still defend the claim that the noun type is universal (Mithun, 2000). Even in languages like Iroquoian, which is sometimes said to have no nouns, there are at least very noun-like words. The adjective type, in contrast, clearly is not universal. If adjectives were phylogenetically earlier than concrete nouns, we should expect the situation with regard to universality be the other way round. In light of the available evidence, proposition P1 is hence relatively well supported, at least if one identifies the contrasting word class with the class of adjectives.

\section{The Structure of Meaning}

One of the main controversies regarding the processing and neuro-cognitive implementation of meaning is whether the semantics of language is processed in a modular or non-modular way. According to modular approaches, the meanings of words and sentences are processed in an informationally largely encapsulated, autonomous, and amodal way (Clifton \& Ferreira, 1987). Candidates for cortical correlates of semantic processes are often supposed to be localized in left temporal and partially frontal regions (Friederici, 2002). Regions typically associated with either perceptual or motor processes in this paradigm are typically not regarded as contributing to semantics. Modular approaches towards perception, in turn, argue for informationally encapsulated, domain-specific and cognitively impenetrable modules for various perceptual tasks (Barrett \& Kurzban, 2006, for review). Modularism with respect to semantics, perception, and perhaps other types of intentional states would thus be hardly compatible with the view that the same mental concept, respectively its neural correlate, is both a meaning provider for linguistic expressions and a content provider for various types of intentional states. A manifold of concept tokens with the content of water would thus be required: the concepts [water]-in-meaning, [water]-in-perception, [water]-in-desire, etc. - eventually even [water]-in-desires-to-drink, [water]-in-desiresto-swim, etc. It is easy to imagine that such a view would quickly lead to an ontological explosion of concepts, at least, if concepts are supposed to exist in a realist manner.

Much more compatible with a realist attitude towards concepts and the methodological goal of ontological parsimony is the anti-modularist view of situated conceptualization (Barsalou, 2005). Here concepts are regarded as situated, i.e., largely based on sensori-motor schemata. The controversy between semantic modularism and semantic anti-modularism relates to the question whether some lexical concepts - i.e., concepts listed in the lexicon and thus expressed by single words - decompose into conceptual parts. Some authors believe that lexical concepts are altogether not decomposable (Fodor \& Lepore, 1992). According to those so-called atomist positions, 
only concepts that are linguistically expressible by syntactically explicitly combined expressions can be complex. In neuroscience some researchers hold that substantial features like that of being an elephant or even features as specific as that of being Halle Berry are represented by highly specialized single neurons (Quian Quiroga et. al., 2005). Lexical atomism is a view semantic modularists can easily live with. For, if meanings are unstructured, it is completely unproblematic to conceive them as localizable elements in an encapsulated module. Proponents of a situated view of meaning, in contrast, will assume that at least some lexical meanings are structured so that parts of the meaning providing concepts may involve various sensori-motor schemata. Semantic anti-modularism seems to exclude lexical atomism.

Our propositions P3 and P4 seem hardly tenable for someone who shares the views of lexical atomism or semantic modularism. Proposition P3 saying that substance concepts expressed by nouns are semantically more complex than concepts expressed by other word classes immediately contradicts lexical atomism, according to which all lexical concepts have the same complexity, viz. zero. Proposition P4 seems to be empty if lexical atomism is true and largely unmotivated if semantic modularism holds. The proposition says that for a cortically implemented syntax-semantics interface, the more widely distributed a concept's neural realization is, the more effort it takes to establish a link between the concept and its lexical expression. Now, if lexical atomism is true, there simply should not be any concepts with a widely distributed neural realization. For, how could this be the case if all lexical concept are unstructured? If semantic modularism were to hold, the meanings even of words that are semantically complex modularism does not entail atomism - would still be locally realized in the postulated semantics module. There would thus be no reason to assume that significantly more effort is needed to assign a word to its meaning, even if the expressed concept is complex.

Since the doctrines of lexical atomism and semantic modularism conflict with P3 and $\mathrm{P} 4$, the natural way to defend the two propositions is to argue against lexical atomism and semantic modularism. This is what I will do in the next section. I will outline a view of situated conceptualization which refutes atomism and modularism.

\section{Situated Conceptualization}

In psychology, philosophy and linguistics various theories have been proposed to account for the decomposition of concepts. For the present purpose the choice of frame theory as a starting point seems most fruitful (Barsalou, 1992). Frame theory provides us with a universal account not only for categorization and its link to action-control, but also for the decomposition of concepts. Frames are recursive attribute-value structures. Attributes assign unique values to objects and thus describe functional relations. The values can be structured frames themselves. A frame is defined for a large domain of things and contains a fixed set of attributes (e.g., color, form) each of which allows for a number of different values (red, green, ... ; round, square, ...). The attributes in question are not constrained to perceptual modalities, but may as well involve attributes of motor affordances. Frames can be nested hierarchically and mutual constraints between attributes (e.g., between states of an object and actions directed to it) and between larger frames can be incorporated (see Figure 1). 


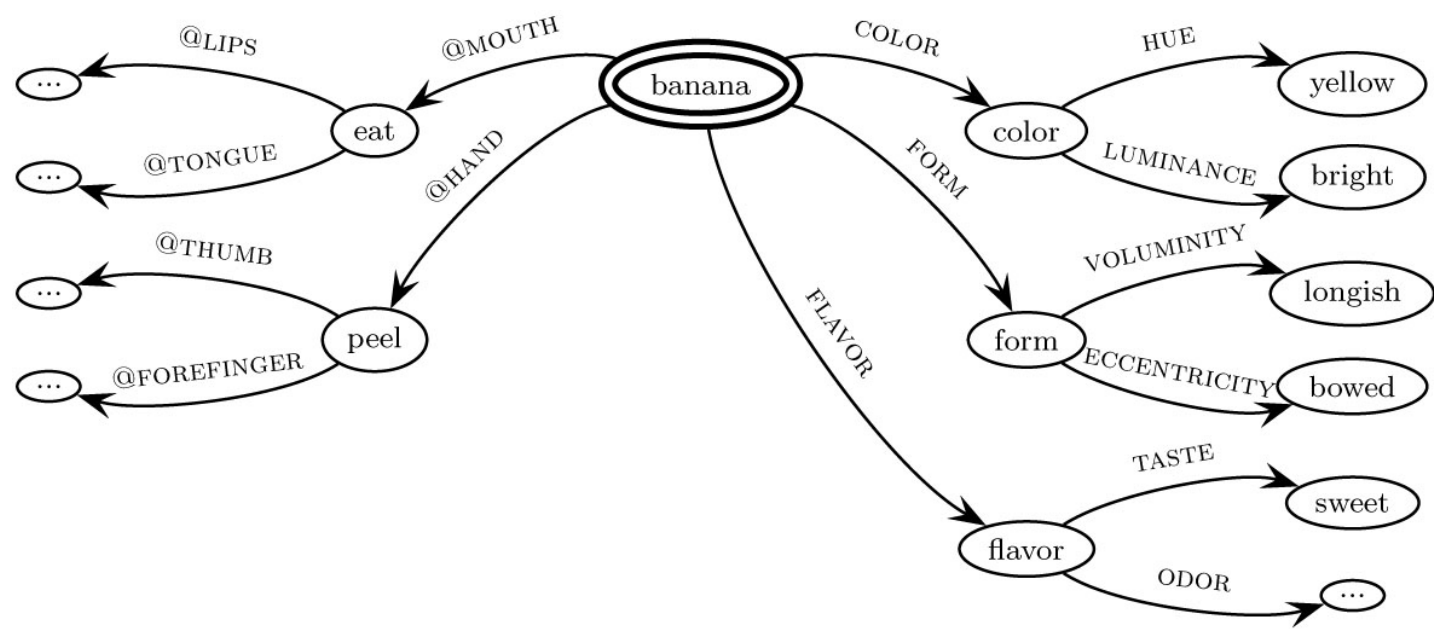

Figure 1. Hypothetical fragment of the frame for the concept [banana]. The substance concept to be decomposed is marked by a double-circle as the referring node of the frame. The labeled arrows denote attributes, the nodes their values. Nodes are themselves regarded as concepts and thus as conceptual parts of the central concept. Whereas, in English, feature attributes (shown on the right) are frequently lexicalized - their arguments typically enter possessive constructions like The color of the banana is yellow or The banana has the color yellow - affordance attributes (on the left) are rarely overtly expressed. Based on linguistic and neurobiological evidence, we assume that affordances often relate to body parts and hence use the convention “@ + body part”. Formally, attributes are mappings from domains of some type into domains of some other type. Petersen \& Werning (2007) provide an explicit account of frames using a calculus of typed feature hierarchies and incorporating typicality effects.

For many attributes involved in perceptual processing one can anatomically identify cortical correlates. Those areas often exhibit a twofold topological structure and justify the notion of a feature map: (i) a receptor topology (e.g., retinotopy in vision, somatotopy in touch): neighboring regions of neurons code for neighboring regions of the receptive field; and (ii) a feature topology: neighboring regions of neurons code for similar features (See Figure 2). With regard to the monkey, more than 30 cortical areas forming feature maps are experimentally known for vision alone (Felleman \& van Essen, 1991). Also affordance attributes seem to have cortical correlates, predominantly in the premotor cortex. The discovery of the so-called mirror neuron system (Rizzolatti. \& Craighero, 2004, for review) may provide a basis to integrate affordances into frames.

The fact that values of different attributes may be instantiated by the same object, but are processed in distinct regions of cortex poses the problem of how this information is integrated in an object-specific way: the binding problem. A prominent and experimentally well supported solution postulates oscillatory neural synchronization as a mechanism of binding: Clusters of neurons that are indicative for different properties sometimes show synchronous oscillatory activity, but only when the properties indicated are instantiated by the same object in the perceptual field; otherwise they are firing asynchronously. Synchronous oscillation, thus, might be regarded to fulfill the task of binding together various property representations to form the representation of an object as having these properties (Singer, 1999). Using oscillatory networks as biologically motivated models, it could be demonstrated how the topological organization of information in the cortex, by mechanisms of synchronization, may yield a logically structured semantics of concepts (Werning, 2005c). Oscillation functions play the role of object concepts. Clusters of feature sensitive neurons play the role of attributive concepts. Schnitzler et al. (2006) could experimentally demonstrate the essential role of neural synchronization for action 


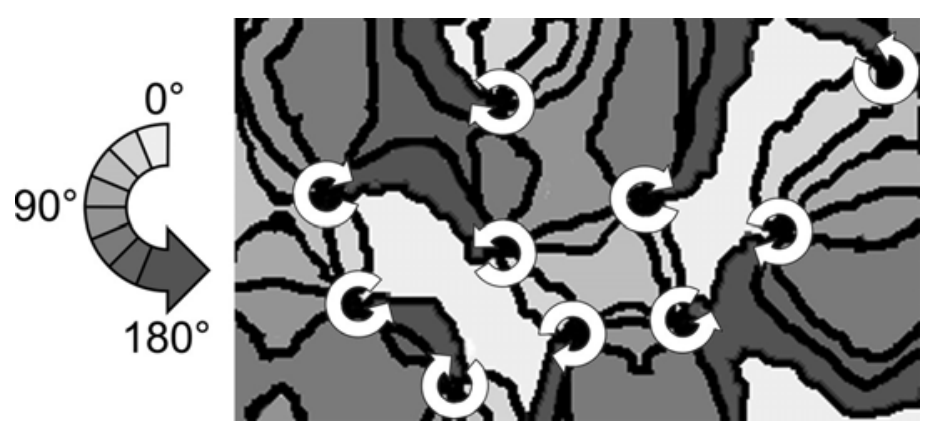

Figure 2. Cortical realizations of frame attributes. Fragment (ca. $4 \mathrm{~mm}^{2}$ ) of the neural feature map for the attribute orientation of cat V1 (adapted from Crair et. al., 1997). The arrows indicate the polar topology of the orientation values represented within each hypercolumn. Hypercolumns are arranged in a retinotopic topology.

control. This may justify the extension of the synchrony-based neuro-frame approach from features to affordances.

Provided that a concept is completely decomposable into a fully specified frame and provided that neural maps for each attribute can be identified in the cortex, the degree to which the cortex represents an object as an instance of the concept is rendered by a general pattern of synchronizing neural activity distributed over neural clusters that correspond to the basic values of the frame. This pattern may be called the cortical fingerprint of the concept.

Support for the theory of neuro-frames also comes from a number of neurolinguistic studies. Based on a review of neurobiological data, Pulvermüller (1999) suggests that neural assemblies that pertain to the sensori-motor cortices and are bound by neural synchronization play an important role in understanding the meanings of words. FMRI studies (Pulvermüller, 2005) regarding the understanding of verbs, e.g., hint to a differential top-down activation of motor and pre-motors areas. We know that the understanding of concrete nouns like hammer, for which not only features, but also affordances are salient, results in an activity distributed over the premotor and the visual cortex (Martin et. al. 1996). The hypothesis that words for substance concepts arouse more widely distributed activity than words for attributive concepts is, furthermore, supported by EEG studies (Rappelsberger et al., 2000).

From this and further evidence (reviewed by Martin, 2007) we may conclude that the correlates of substance concepts are highly distributed neural states. Substance concepts are thus not expected to be realized by single cells, or locally circumscribed regions of the cortex, but by cell assemblies that may pertain to highly distinct parts of the cortex and involve perception as well as motor areas. In contrast, the neural correlates of attributive concepts would be constrained to local cortical regions. The view that substance concepts decompose into complex frames and that their neural realizations are widely distributed in cortex contradicts the doctrines of atomism and modularism.

\section{Evolution and Development of the Syntax-semantics Interface}

Another strategy to avoid the paradox is to limit the scope of the assumption P2 that the meanings of concrete nouns are substance concepts. One might advocate a meaning shift of a certain kind in nouns during development or evolution: Whereas for modern adults concrete nouns express substance concepts with a complex semantics, it might be that the child's usage of the noun mama only labels a salient person in his or her daily 
life or that, for an early human, the noun for water just expressed the affordance of being drinkable. It is indeed very likely that the concepts expressed by nouns change in development and evolution. [birth-giving] is not a conceptual part of [mama] for the two-year old as it is for us. Early humans did not represent water as molecularly complex. However, is it plausible that nouns of young children and early humans do not at all express substance concepts with some decent, if only different, semantic complexity? How could the word mama in the child's language be a label for a particular person if the child were not able to recognize and treat that person as mama (in his/her sense)? To recognize and treat mama as mama - we have to postulate - the child mentally represents a number of salient features and affordances. Otherwise we would have to withdraw to a rather unwarranted iconic theory of representation.

In the case of phylogeny, the challenge could also be phrased as follows: Was there a time when [water] was an attributive concept - for a simple affordance or feature? That substance concepts finally reduce to one (or a small number) of attributive concepts is the tenet of essentialism: If essentialism about conceptual representation is true, we represent a substance by few essential features or affordances which the substance must never change. The problem is that for most everyday substances one can hardly find any cognitively plausible candidates for essences. Being $\mathrm{H}_{2} \mathrm{O}$ is essential for water, but is this how humans cognitively represent water? The alternative is to decompose a substance concept into a structure of feature and affordance concepts, none of which specifies an essential property, but only a typical one. Even though water prototypically is tasteless, there is salty water. Water can change its color, taste, aggregate state, etc., even though some values for each of those attributes are more typical than others. Water is also used in typical ways: for drinking, washing, swimming, but it can also be burned by magnesium torches.

There are, of course, lots of nouns in English that express single attributive concepts: abstract nouns. The large majority of them are morphologically derived or, at least, syntactically marked (compare water to beverage, fluidity, etc.). This indicates that nouns expressing single attributive concepts are evolutionarily rather late. There is thus little evidence that [water] in the early stages of language evolution ever was a semantically simple attributive concept, rather than a semantically complex substance concept as it is today. P2 holds also for the early stages of development and evolution.

The remaining option to attack the paradox seems to be the principle P5 that capabilities demanding more effort, ceteris paribus, develop and, respectively, evolve later than those demanding less effort. One might argue that the demand of effort is not the only, maybe not even the most important factor that determines evolutionary priority. One may point out that there is stronger evolutionary pressure to lexicalize concepts as complex as substance concepts than to lexicalize attributive concepts. It arguably is rather economic to lexicalize concepts for often recurring, highly specific entities of great survival value. Telling someone that there are bananas somewhere is not only shorter, but also more exact than telling someone that there are sweet, longish, bowed, bright, yellow things around that one may peel and eat. However, an appeal to greater selection pressure does not suffice to explain evolutionary priority: To explain why proto-birds evolved wings, one has to appeal to some sort of evolutionary pressure to fly. If flying did not have a selective advantage for proto-birds, wings would not have evolved. Maybe proto-birds had to reach or leave trees quickly to escape predators. However, if proto-birds had not had feathers in the first place (maybe for cooling as some hypothesis goes), wings would not have evolved either. Even if selection pressure were maximal and flying the only way a certain reptile species could have survived, if the species did not have feathers and very wing-like forelimbs, it would have died out rather than evolve wings. In addition to evolutionary pressure any explanation of 
capabilities must appeal to some step-by-step evolution of mechanisms: from the more primitive to the more complex.

What we still have no answer for is the following question: How could a mechanism evolve that enables certain regions of cortex that are involved in representing a word (phonologically, syntactically, etc.) to address those regions of the sensori-motor cortices that represent the word's meaning, i.e., the concept it expresses. Given that semantically complex words are evolutionary prior such an interface must have had strong distributive capacities from the beginning.

\section{References}

Arbib, M. A. (2005). From monkey-like action recognition to human language: An evolutionary framework for neurolinguistics. Behavioral and Brain Sciences, 28(2), 105-24.

Barrett, H., \& Kurzban, R. (2006). Modularity in cognition. Psychological Review, 113, 628-47.

Barrett, M. (1995). Early lexical development. In P. Fletcher \& B. MacWhinney (Eds.), The handbook of child language (pp. 362-92). Cambridge, MA: Blackwell.

Barsalou, L. (1992). Frames, concepts, and conceptual fields. In A. Lehrer \& E. Kittay (Eds.), Frames, fields, and contrasts (pp. 21-74.). Hillsdale, NJ: Erlbaum.

Barsalou, L. (2005). Situated conceptualization. In H. Cohen \& C. Lefebvre (Eds.), Handbook of categorization in cognitive science (pp. 619-50). St. Louis: Elsevier.

Bloom, P. (2000). How children learn the meanings of words. Cambridge, MA: MIT Press.

Boas, F., \& Deloria, E. (1939). Dakota grammar. Memoirs of the National Academy of Sciences, 23(2).

Clifton, C., \& Ferreira, F. (1987). Modularity in sentence comprehension. In J. Garfield (Ed.), Modularity in knowledge representation and natural-language understanding (pp. 277-90). MIT Press.

Crair, M., Ruthazer, E., Gillespie, D., \& Stryker, M. (1997). Ocular dominance peaks at pinwheel center singularities of the orientation map in cat visual cortex. Journal of Neurophysiology, 77, 3381-5.

Crowley, T. (1995). Inalienable possession in Paamese grammar. In H. Chappell \& W. McGregor (Eds.), The grammar of inalienability (pp. 383-432). Berlin: de Gruyter.

Dixon, R. (1999). Adjectives. In K. Brown, J. Miller, \& R. Asher (Eds.), Concise encyclopedia of grammatical categories (pp. 1-8). Amsterdam: Elsevier.

Felleman, D., \& van Essen, D. (1991). Distributed hierarchical processing in the primate cerebral cortex. Cerebral Cortex, 1, 1-47.

Fodor, J. (1992). A theory of content and other essays. Cambridge, MA: MIT Press.

Fodor, J., \& Lepore, E. (1992). Holism: A shopper's guide. Oxford: Blackwell.

Friederici, A. (2002). Towards a neural basis of auditory sentence processing. Trends in Cognitive Sciences, 6, 78-84.

Givon, T. (1970). Notes on the semantic structure of English adjectives. Language, 46, 816-37.

Goldfield, B., \& Reznick, J. (1990). Early lexical acquisition: rate, content, and the vocabulary spurt. Journal of Child Language, 17, 171-83.

Ingram, D. (1989). First language acquisition; method, description and explanation. Cambridge: CUP.

Martin, A. (2007). The representation of object concepts in the brain. Annual Review of Psychology, 58, 25-45. 
Martin, A., Wiggs, C. L., Ungerleider, L., \& Haxby, J. V. (1996). Neural correlates of category specific knowledge. Nature, 379, 649-52.

Millikan, R. G. (1998). A common structure for concepts of individuals, stuffs and real kinds: More mama, more milk, and more mouse. Behavioral and Brain Sciences, 21, 55-100.

Mithun, M. (2000). Noun and verb in Iroquoian languages: Multicategorisation from multiple criteria. In P. Vogel \& B. Comrie (Eds.), Approaches to the typology of word classes (pp.397-420). Berlin: de Gruyter.

Petersen, W., \& Werning, M. (2007). Conceptual fingerprints: Lexical decomposition by means of frames - a neuro-cognitive model. In U. Priss, S. Polovina, \& R. Hill (Eds.), Conceptual structures: Knowledge architectures for smart applications (LNAI 4604) (pp.415-28). Heidelberg: Springer.

Pulvermüller, F. (1999). Words in the brain's language. Behavioral and Brain Sciences, 22, 253-79.

Pulvermüller, F. (2005). Brain mechanisms linking language and action. Nature Reviews Neuroscience, 6, 576-82.

Quian Quiroga, R., Reddy, L., Kreiman, G., Koch, C., \& Fried, I. (2005). Invariant visual representation by single-neurons in the human brain. Nature, 435, 11027.

Rappelsberger, P., Weiss, S., \& Schack, B. (2000). Coherence and phase relations between EEG traces recorded from different locations. In R. Müller (Ed.), Time and the brain (pp. 297-330). Harwood.

Rizzolatti, G., \& Craighero, L. (2004). The mirror-neuron system. Annual Review of Neuroscience, 27, 169-92.

Schnitzler, A., Timmermann, L., \& Gross, J. (2006). Physiological and pathological oscillatory networks in the human motor system. Journal of Physiology, 99, 3-7.

Singer, W. (1999). Neuronal synchrony: A versatile code for the definition of relations? Neuron, 24, 49-65.

Werning, M. (2005a). The 'complex first' paradox: What's needed to compose neurally distributed lexical meanings? Behavioral and Brain Sciences, 28(2) (online suppl.).

Werning, M. (2005b). Right and wrong reasons for compositionality. In M. Werning, E. Machery, \& G. Schurz (Eds.), The compositionality of meaning and content (Vol. I: Foundational Issues, pp. 285-309). Frankfurt: Ontos Verlag.

Werning, M. (2005c). The temporal dimension of thought: cortical foundations of predicative representation. Synthese, 146, 203-24.

Werning, M. (2008). The 'complex first' paradox: Why do semantically thick concepts so early lexicalize as nouns? Interaction Studies, 9, 67-83.

Address of correspondence:

Department of Philosophy

Heinrich-Heine University Düsseldorf

Universitätsstr. 1

40225 Düsseldorf

Germany

E-mail:

werning@phil.uni-duesseldorf.de 